\title{
Manner of speech and its influence on speech understanding in older patients with impaired hearing
}

\author{
Raymond H Hull* \\ Department of Communication Sciences and Disorders, College of Health Professions, Wichita State University, Wichita, Kansas, USA
}

One of the consistent complaints heard from our older adult patients is, "I can hear, but I can't understand what people are saying!" There can be several reasons for that complaint, but among those the most common source of the problem can involve the speaking habits of many adults. As I tell my patients, "Hearing aids cannot improve the speech of those who do not speak clearly". Even for those with hearing that is considered normal, unless the listening environment is optimal, the speech of some adults can be difficult to understand. This paper describes the problem of speech understanding in older adulthood and a method for enhancing the ability of older adults to understand spoken speech with greater ease in spite of a sensorineural hearing loss and a possible decline in central auditory processing, both of which generally reveal themselves in advancing age.

Barring physical impairments that can negatively influence speech clarity, there are many causes that can result in speech produced by speakers that is difficult to understand. Among those reasons include speech that is spoken too rapidly which results in a lack of precision of articulation, lack of vocal intensity and control, lack of the suprasegmental components (the melody and inflections) of speech, physical barriers that impede the speech signal, among others. And to compound those factors, besides having to cope with the probability of a sensorineural hearing loss at the peripheral level in older age that can result in difficulty recognizing important phonemes of speech that can impair speech understanding, there is also the possibility of a central auditory decline with aging that can impede the speed and precision of the processing of spoken speech.

A primary reason that a combination of both of those detrimental aspects of an aging auditory system can impair one's ability to understand spoken speech centres on the fact that spoken speech involves a complex combination of voiced and voiceless phonemes that are easily distorted during their utterance. They are combined in countless ways to form words that are combined to form sentences that are sometimes uttered in abbreviated forms. Speech that is uttered too rapidly can result in sentences that become akin to elongated multisyllabic words that make speech understanding even more difficult.

The gymnastics required of the peripheral and central auditory systems (cochlea, brainstem and association areas of the brain) as the older adult attends to and processes spoken information throughout each day are enormous. In order to process speech and language, the individual's central nervous system must be involved in an extremely complex set of neurologic events. Not only does the central nervous system have to accurately receive and translate a complex phonemic/ suprasegmental/linguistic/neurochemically-based code that we call speech, it must do so at an extremely rapid rate in order to process and comprehend ongoing spoken speech. While doing so, it must be able to compare the informational concepts that it is receiving with previously learned and stored vocabulary that has been housed within the central nervous system, and then determine its content and meaning along with the semantic intent of the message. This is an extremely complex set of acoustic/neurologic/linguistic events that must be completed accurately nearly within milliseconds, if not microseconds in order to keep up with what is being said by speakers who may be speaking at 180 words per minute.

This is a great challenge for older adults who possess no disabilities within their peripheral auditory system, but the central auditory system may have slowed to some degree. But, it is an even greater challenge for older adults who possess a sensorineural hearing loss that may deprive the listener from hearing important high frequency phonemes of speech, and also a possible decline in central auditory processing that can impair the person's ability to process and comprehend what is received with the speed and accuracy that the person once enjoyed.

\section{What can be done to assist these older patients?}

What can be done to assist these older patients? As I mentioned earlier, hearing aids cannot improve the speaking habits of those who do not speak clearly, nor do they improve a central auditory processing system that has declined in its ability to process speech with the speed and accuracy necessary to interpret rapidly spoken speech.

\section{First-there is a problem that must be overcome}

First of all, there is a problem that must be overcome. Early research by Pichney et al. [1,2] and Krause et al. [3], among others on the topic of the characteristics of clear speech and its impact on speech intelligibility for older adults, and our unpublished research completed at this University utilizing time compressed speech have all shown that speech that is spoken at a slower than average rate is consistently clearer and more understandable for older adults, including those with impaired hearing. For example, adults at age 75 years can comprehend the content of spoken speech with consistency when it is spoken at a rate of around 124 words per minute (WPM) [4]. Therefore, it is particularly troublesome for older adults with impaired auditory function when typical adult speech is generally spoken at between 160 to $180 \mathrm{WPM}$ [4]. And, when this author has been asked to work with television news and sports broadcasters in situations where complaints have been

*Correspondence to: Raymond H Hull, Department of Communication Sciences and Disorders, College of Health Professions, Wichita State University, Wichita, Kansas, USA, Tel: +1 316-978-3456; E-mail: Ray.Hull@wichita.edu

Received: September 02, 2019; Accepted: September 16, 2019; Published: September 20, 2019 
received because older adults have had difficulty understanding their news broadcasts, he has timed those broadcasters at speeds of spoken speech exceeding 190 WPM. It becomes nearly impossible for older adults who may have a combination of both peripheral hearing loss and central auditory decline to understand speech that is spoken at that rate.

That could be a reason older adult tell this author that they appreciated former news broadcasters such as Walter Cronkite and Tom Brokaw who spoke during their news broadcasts at rates in the vicinity of 128 to $130 \mathrm{WPM}$. They could understand what they were saying with some ease.

So, that is the problem that must be overcome. Since the average rate of spoken speech by adults (in the U.S.) appears to be somewhere in the vicinity of 160 to $180 \mathrm{WPM}$, it becomes difficult for older adults to process what is being said with the speed and accuracy necessary to comprehend what other adults are saying. Sentences tend to become lengthy multisyllabic words, such as "Duyawannavlnch?" or "Wergongtuthestortulukfershuz" when the intent was to say, "Do you want to have lunch?" or "We're going to the store to look for shoes". With the possibility of a high frequency sensorineural hearing loss that may restrict the audibility of high frequency consonant phonemes that may also accompanied by a central auditory system that has declined in its ability to process rapidly spoken speech with speed and accuracy, it becomes difficult for an older adult to understand the typical speech of adults who may be communicating with them.

Second--beside wearing hearing aids that may only amplify the poor speaking habits of many adults, what can be done to improve speech understanding for older adults who may possess both peripheral and central auditory impairments?

One important thing that can be done includes encouraging those who frequently speak with older adults with impaired hearing to practice speaking at a slower rate that will allow the older peripheral and central auditory systems to function with greater efficiency. Those include family members, ministers, and other significant others in the life of the older adult.

\section{Practice-practice--practice}

One suggestion for purposes of practice speaking at a slower rate is to obtain a DVD or other recording of Mr. Roger's Neighbourhood. Fred Rogers spoke at a rate of around 126 WPM which is a perfect rate for speech comprehension by both young children and older adults. The DVD can be used to mimic the rate of speech of Fred Rogers, and practice speaking at his rate of speech that is probably slower than one's usual rate.

Further, a DVD or other recording of the former news commentators Walter Cronkite or Tom Brokaw would be useful for the purpose of practice. All three of the personalities mentioned here rehearsed speaking at a rate of around 124-126 WPM. Those who communicate with older adults can practice mimicking the rate of speech on those recordings, and then rehearsing until it becomes habitual.

\section{Another avenue for practice}

Another activity that can be engaged in involves finding a paragraph to read either out of a favourite book, a magazine such as
Readers Digest, or a children's book--in other words, anything that is easy to read aloud. Choose any paragraph and count 124 words, then place a mark after the $124^{\text {th }}$ word. Locate a stopwatch or any watch or clock with a second hand. It is helpful for someone to assist with this activity. Have that person tell you when to begin reading. At "go", the paragraph is read at the reader's normal rate of speech. It must be read aloud to mimic spoken speech. The "time keeper" tells the reader when 60 seconds are up. If the reader finishes the 124 word paragraph(s) before one minute is up, then speech is being uttered too rapidly. This activity should to be repeated until the "speaker" is consistently completing the 124-word paragraph at the same moment one minute is up. It will probably seem to the reader that she or he is reading (speaking) much too slowly. However, that is the rate of speech at which older adults, and I should add most children and most adults will understand best. And, that should be the speaking rate one rehearses to become habitual.

\section{The Result?}

If that rate of speech is used when speaking to older patients, they will be amazed at how well they can understand what is being said. For family members, physicians, nurses, preachers, and all who communicate with older adults, it should become the norm rather than the exception that older adults and those who communicate with them will recognize that they have become easier to listen to and understand when speaking rate is slowed. Family members will also find that the older members of their family are understanding what they are saying without having to be asked to repeat so often. Life for all generally becomes less frustrating. And, it can be a gratifying experience!

\section{An added benefit of a slower rate of speech}

There is an added benefit to speaking at a slower rate. As one reduces the speed of their spoken speech, precision of articulation is naturally enhanced. The reason is that when speech is spoken at a slower rate, the vowel and consonant sounds are elongated and therefore spoken in their complete form as they are meant to be. Additionally, the melody and inflections of speech are naturally enhanced. Whole words are spoken, "sentences" become actual sentences formed with whole words, meaningful pauses, and the melody and inflection that enhances speech understanding, as opposed to the elongated sets of partial words and sounds that come from rapid speech. It greatly enhances speech understanding for older adults (and, of course, young children too, and virtually all adults!).

We can all speak with greater clarity if we just slow down!!

\section{References}

1. Picheny M, Durlach N, Braida L (1985) Speaking clearly for the hard of hearing. $J$ Speech Language Hearing Res 26: 96-103.

2. Picheny M, Durlach N, Braida, L (1989) Speaking clearly for the hard of hearing III. $J$ Speech Language Hearing Res 32: 600-603.

3. Krause J, Braida L (2002) Investigating alternative forms of clear speech: The effects of speaking rate and speaking mode on intelligibility. J Acoust Soc Am 112: 2165-2171. [Crossref]

4. Robinson H, Hull R (2015) Effect of speed of speech presentation on speech comprehension in adults. Unpublished Doctor of Audiology Research. Wichita, KS Wichita State University.

Copyright: (C2019 Zhu P. This is an open-access article distributed under the terms of the Creative Commons Attribution License, which permits unrestricted use, distribution, and reproduction in any medium, provided the original author and source are credited. 